F.-W. Riffelmann

G. Droste

H. Lauter

D. Köhler

\title{
Untersuchung zum Karenzintervall von Montelukast vor inhalativer bronchialer Allergenprovokation
}

\author{
Montelukast-Free Interval Before Inhalational Bronchial Allergen Provocation
}

\section{Zusammenfassung}

Hintergrund: Leukotriene besitzen als Mediatoren bei Asthma bronchiale eine starke bronchokonstriktorische und proinflammatorische Potenz. In einer Voruntersuchung konnten wir an 16 Probanden mit Asthma bronchiale (2 Gruppen, randomisiert) zeigen, dass Montelukast im Vergleich zum Leerversuch über 21 Tage (10 mg/d) die Allergenprovokation mit dem jeweiligen spezifischen Allergen (Milben und Pollen) deutlich hemmt $(p=0,0005)$. In einer Anschlussuntersuchung wollten wir der Frage nachgehen, wie lange Montelukast abgesetzt werden muss, um einen relevanten Einfluss auf die inhalative bronchiale Allergenprovokation zu vermeiden. Methode: Montelukast wurde am Ende der ersten Studie nochmals über 21 Tage $(10 \mathrm{mg} / \mathrm{d})$ in den beiden Gruppen $(2 \times 8$ Pat. mit Asthma) verabreicht. Nach einer Pause von 1 bzw. 3 Tagen wurde die Allergenprovokation in identischer Weise wiederholt und mit den historischen Ausgangswerten ohne Montelukast verglichen. Ergebnisse: Die Provokationsdosis für Allergen reduzierte sich nach 1 Tag Einnahmepause um 39\%, nach 3 Tagen um 67\%. Nur nach 3 Tagen war der Unterschied signifikant ( $p=0,017$ ) und es wurde fast wieder der Leerwert erreicht. Schlussfolgerung: Wir empfehlen, die Medikation mit Montelukast mindestens 3 Tage vor inhalativer Allergenprovokation abzusetzen, da sich erst ab dann die protektive Wirkung von Montelukast verliert.

\section{Abstract}

Background: Leukotrienes are potent bronchoconstrictive and proinflammatory mediators in bronchial asthma. In a previous study on 16 subjects with bronchial asthma (two randomized groups) we showed that montelukast (10 mg daily) significantly suppressed allergen provocation with specific allergens (mites and pollen) ( $p=0.0005)$. In a follow-up study we addressed the question as to when montelukast should be discontinued in order to avoid a relevant effect on inhalational bronchial allergen provocation. Method: At the end of the first study montelukast (10 mg daily) was given again to both groups ( $2 \times 8$ asthma patients) for 21 days. After a montelukast-free period of one and three days allergen provocation was repeated in the same manner and the results were compared with the historic baseline values without montelukast. Results: The provocation dose for allergens was reduced by $39 \%$ after a montelukast-free period of one day and by $67 \%$ after three days. Only after three days was the difference significant $(p=0.017)$, at which time the control value was nearly reached again. Conclusion: We recommend that montelukast be discontinued at least three days prior to inhalational allergen provocation. 
Asthma bronchiale ist eine chronisch entzündliche Atemwegserkrankung, charakterisiert durch eine Infiltration der Bronchialschleimhaut durch Entzündungszellen (Lymphozyten, eosinophile Granulozyten und Mastzellen), verbunden mit der Produktion verschiedener Mediatoren [1].

Unter den Mediatoren besitzen die Leukotriene eine starke bronchokonstriktorische und proinflammatorische Wirksamkeit, sie steigern die bronchiale Hyperreagibilität sowie die Mukussekretion und erhöhen die Gefäßpermeabilität $[2,3]$.

Die über verschiedene Zwischenschritte aus der Arachidonsäure gebildeten Cysteinyl-Leukotriene (CystLT) binden an einen gemeinsamen Rezeptor, der als CystLT $_{1}$-Rezeptor bezeichnet wird [4]. Montelukast (Singulair ${ }^{\circledR}$ ) ist als erster spezifischer CystLT $_{1}$-Rezeptorantagonist seit 1998 zur Behandlung des Asthma bronchiale in Deutschland im Handel (Herstellerangabe Fa. MSD Dieckmann, Haar).

In vielen Publikationen hat sich Montelukast als effektive Substanz zur Reduktion der asthmaassoziierten bronchialen Entzündung erwiesen [1,5-7].

In einer Voruntersuchung [8] konnten wir zeigen, dass eine Vormedikation mit dieser Substanzklasse einen relevanten Einfluss auf die inhalative bronchiale Allergenprovokation hat, die zur Abschätzung der klinischen Relevanz einer kutanen Sensibilisierung häufig unerlässlich ist. Bislang existieren keine Daten, die Auskunft über die erforderliche Absetzfrist vor Allergenprovokation geben.

Deshalb führten wir eine randomisierte Studie durch, in der der Einfluss einer Unterbrechung der Einnahme von Montelukast (1 bzw. 3 Tage) auf die inhalative bronchiale Allergenprovokation untersucht wurde. Es handelt sich um eine Fortsetzung der von uns bereits publizierten Untersuchung über Montelukast und inhalative Allergenprovokation [8], so dass auf Daten aus dieser Untersuchung zurückgegriffen wird.

\section{Methode}

\section{Patienten}

Die Untersuchung erfolgte an insgesamt 16 Probanden mit Asthma bronchiale in 2 Gruppen (randomisiert). Die Einschlusskriterien und die biometrischen Daten sind in Tab. 1 und 2 aufgeführt.

\section{Untersuchungsablauf}

Nach Abschluss der Voruntersuchung erfolgte in Gruppe I für weitere 21 Tage eine Medikation mit Montelukast, $10 \mathrm{mg} / \mathrm{d}$ (Abb.1). Nach 1 Tag therapiefreiem Intervall wurde am 23. Tag eine erneute inhalative bronchiale Allergenprovokation durchgeführt (Details s. u.). In der zweiten Gruppe erfolgte ebenfalls eine 21-tägige Montelukast-Therapie; die Allergenprovokation wurde allerdings erst nach einem therapiefreien Intervall von 3 Tagen, also am 25. Tag durchgeführt.
Tab 1 Begleitmedikation und Einschlusskriterien

\begin{tabular}{|c|c|c|}
\hline $\begin{array}{l}\text { Begleitmedikation } \\
\text { erlaubt }\end{array}$ & verboten & Einschlusskriterien \\
\hline inhalative Kortikosteroide & orale Kortikosteroide & $\mathrm{FEV}_{1}>70 \%$ des Sollwertes \\
\hline $\begin{array}{l}\text { kurzwirksame } \\
\beta \text {-Mimetika }\end{array}$ & $\begin{array}{l}\text { langwirksame } \\
\beta \text {-Mimetika }\end{array}$ & $\begin{array}{l}\text { Vorliegen eines Asthma } \\
\text { bronchiale mit }\end{array}$ \\
\hline \multirow[t]{5}{*}{ Anticholinergika } & orale $\beta$-Mimetika & $\begin{array}{l}\text { Nachweis einer unspez. } \\
\text { bronch. Hyperreagibilität }\end{array}$ \\
\hline & Theophyllin & Alter > 18 Jahre \\
\hline & $\begin{array}{l}\text { system. Antihista- } \\
\text { minika }\end{array}$ & $\begin{array}{l}\text { Nachweis einer klinisch } \\
\text { relevanten aktuellen }\end{array}$ \\
\hline & DNCG/Nedocromil & $\begin{array}{l}\text { Sensibilisierung mit } \\
\text { perennialen oder }\end{array}$ \\
\hline & $\beta$-Blocker & saisonalen Allergenen \\
\hline
\end{tabular}

Tab. 2 Basischarakteristika

\begin{tabular}{|c|c|c|c|c|}
\hline Gruppe I & Alter & $m / w$ & $\mathrm{FEV}_{1}(\% S W)$ & BHR \\
\hline 1 & 19 & w & 90 & 38 \\
\hline 2 & 27 & $\mathrm{~m}$ & 87 & 9 \\
\hline 3 & 30 & $\mathrm{~m}$ & 78 & 140 \\
\hline 4 & 40 & w & 116 & 70 \\
\hline 5 & 37 & $\mathrm{~m}$ & 80 & 50 \\
\hline 6 & 40 & $\mathrm{~m}$ & 70 & 10 \\
\hline 7 & 26 & w & 102 & 160 \\
\hline 8 & 51 & w & 118 & 240 \\
\hline MW & 33,8 & & 92,6 & 89,6 \\
\hline$S D$ & 10,2 & & 17,7 & 82,4 \\
\hline \multicolumn{5}{|l|}{ Gruppe II } \\
\hline 1 & 24 & $\mathrm{~m}$ & 94 & 180 \\
\hline 2 & 58 & $\mathrm{~m}$ & 91 & 96 \\
\hline 3 & 28 & w & 70 & 14 \\
\hline 4 & 26 & w & 91 & 9 \\
\hline 5 & 28 & $\mathrm{~m}$ & 89 & 20 \\
\hline 6 & 23 & $\mathrm{~m}$ & 72 & 15 \\
\hline 7 & 33 & w & 102 & 10 \\
\hline 8 & 43 & $\mathrm{~m}$ & 96 & 120 \\
\hline MW & 32,9 & & 88,1 & 58 \\
\hline$S D$ & 12,0 & & 11,3 & 65,6 \\
\hline
\end{tabular}

BHR (unspezifische bronchiale Hyperreagibilität)

Angegeben als $\mathrm{PD}_{20 \mathrm{Histamin}}$ in $\mu$ intrabronchial deponierter Histaminmasse

Die übrige vorbestehende Medikation wurde während des gesamten Untersuchungszeitraums nicht verändert; die erlaubte bzw. verbotene Begleitmedikation ist in Tab.1 zusammengestellt.

\section{Methodik der Allergenprovokation}

Die Allergenverneblung erfolgte mithilfe eines Düsenverneblers (Fa. Pari, Starnberg) mit TIA S-Inhalierkopf und IS 2-Kompressor, der ein Aerosolspektrum erzeugt, welches - wie bei der Allergenprovokation gewünscht - überwiegend in den zentralen Atemwegen deponiert (mittlerer aerodynamischer Massendurchmesser 8,5 $\mu \mathrm{m}$ ) (Herstellerangabe Fa. Pari, Starnberg). 
Voruntersuchung (8)

aktuelle

Untersuchung

zur Absetzfrist

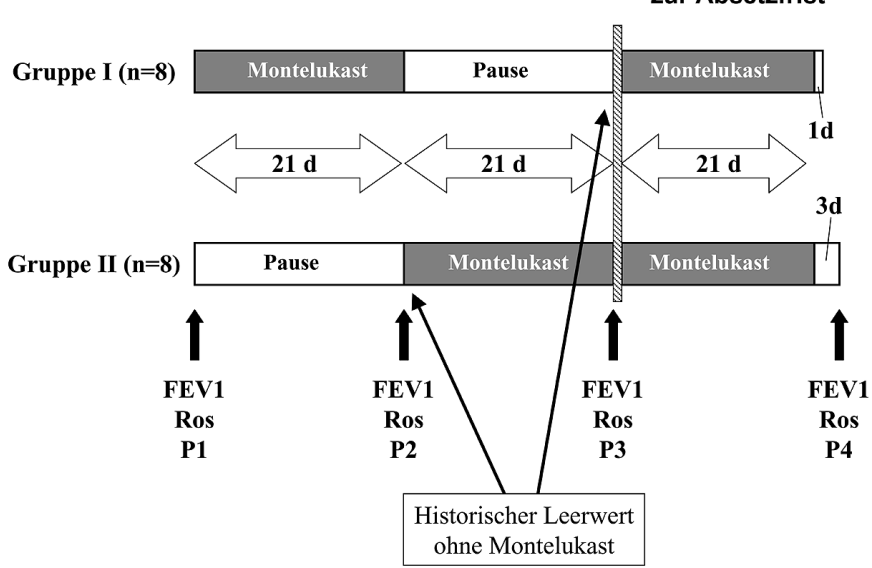

Abb. 1 Untersuchungsablauf

$\mathrm{P}=$ Provokationstest mit Allergen, Ros = oszillatorische Resistance

Es wurden handelsübliche Provokationslösungen (Fa. Allergopharma, Reinbek) verwendet in der Verdünnung: $1: 1000$, $1: 100,1: 50,1: 10$ und $1: 5$.

Alle 20 Minuten wurde die Allergenkonzentration gesteigert, sofern die Positivkriterien (Abfall der $\mathrm{FEV}_{1}$ um 20\% oder Verdopplung der oszillatorischen Resistance) noch nicht erfüllt waren. Die Messung der FEV F $_{1}$ erfolgte mit dem Asthma-Monitor der Fa. Jaeger; die Bestimmung der oszillatorischen Resistance mit dem Siregnost FD 5 der Fa. Siemens. Die Allergenprovokation erfolgte in Übereinstimmung mit den neuesten Leitlinien für die inhalative bronchiale Allergenprovokation der pneumologischen und allergologischen Fachgesellschaften [9]. Um für eine statistische Analyse verwertbare Daten zu erhalten, war es erforderlich, die inhalierte Allergenmenge zu quantifizieren. $1 \mathrm{ml}$ eingesetzter $\mathrm{Al}$ lergenextrakt enthält unverdünnt $5000 \mathrm{BE} / \mathrm{ml}(\mathrm{BE}=$ biologische Einheiten).

In Kenntnis der Literatur darf man davon ausgehen, dass mit dem oben genannten Inhalationssystem im Mittel ca. 15\% der eingesetzten Allergenmenge intrabronchial deponiert wird [10], somit pro $\mathrm{ml}$ ca. $750 \mathrm{BE}$. Die gemessenen $\mathrm{FEV}_{1}$-Werte wurden auf logarithmischem Papier in Bezug zur inhalierten Allergenmenge der jeweiligen Verdünnungsstufe gesetzt. Die Allergenmenge, bei der eine $20 \%$ ige Reduktion des $\mathrm{FEV}_{1}$ bzw. eine Verdopplung der Ros auftrat, wurde wie üblich als $\mathrm{PD}_{20 \text { Allergen }}$ bezeichnet und war Ausgangspunkt für die Berechnungen.

\section{Statistik}

Die Unterschiede im Zeitverlauf mit und ohne Montelukast wurden durch den Wilcoxon-Test für verbundene Stichproben auf Signifikanz geprüft wobei ein $\mathrm{p}$-Wert $\leq 0,05$ als signifikant galt.

\section{Ergebnisse}

Die biometrischen Daten beider Gruppen waren nicht signifikant verschieden (s. Tab. 2). Zwischen der Provokation unter Montelukast und Ausgangswert findet sich in beiden Gruppen zusam-
Tab. 3 Messwerte in BE (Biologiche Einheiten) und Allergene

\begin{tabular}{|c|c|c|c|c|}
\hline \multirow[t]{2}{*}{ Gruppe I } & $\begin{array}{l}\text { Ausgangswert } \\
\text { (P1) }\end{array}$ & $\begin{array}{l}\text { unter Monte- } \\
\text { lukast (P2) }\end{array}$ & $\begin{array}{l}1 \text { Tag Pause } \\
\text { (P3) }\end{array}$ & \multirow[t]{2}{*}{ Allergen } \\
\hline & PD20-Allergen & PD20-Allergen & PD20A-Ilergen & \\
\hline 1 & 150 & 130 & 150 & Milbe d.pt. \\
\hline 2 & 2 & 1,3 & 1,3 & Milbe d.f. \\
\hline 3 & 11 & 18 & 1,2 & Katze \\
\hline 4 & 3,5 & 75 & 15 & Milbe d.f. \\
\hline 5 & 11 & 85 & 45 & Milbe d.pt. \\
\hline 6 & 10 & 6 & 9 & Milbe d.f. \\
\hline 7 & 3,5 & 28 & 19 & Milbe d.f. \\
\hline 8 & 8 & 75 & 15 & Erle \\
\hline Mittelwerte & 16,58 & 52,29 & 31,94 & \\
\hline$S D$ & 50,68 & 45,75 & 49,66 & \\
\hline Gruppe II & $\begin{array}{l}\text { Ausgangswert } \\
\text { PD20-Allergen }\end{array}$ & $\begin{array}{l}\text { unter Monte- } \\
\text { lukast } \\
\text { PD20-Allergen }\end{array}$ & $\begin{array}{l}3 \text { Tage Pause } \\
\text { PD20-Allergen }\end{array}$ & \\
\hline 1 & 33 & 300 & 120 & Milbe d.f. \\
\hline 2 & 1,4 & 12 & 4,5 & Milbe d.f. \\
\hline 3 & 1,8 & 12 & 2,6 & Hasel \\
\hline 4 & 4 & 11 & 6 & Milbe d.pt. \\
\hline 5 & 11 & 30 & 11 & Gräser \\
\hline 6 & 9 & 7,5 & 8 & Milbe d.pt. \\
\hline 7 & 7,5 & 15,50 & 2 & Milbe d.pt. \\
\hline 8 & 12 & 120 & 14 & Hasel \\
\hline Mittelwerte & 9,96 & 63,5 & 21,01 & \\
\hline$S D$ & 10,13 & 102,64 & 40,20 & \\
\hline
\end{tabular}

$\mathrm{P}=$ Provokationstest

d. f. = Dermatophagoides farinae, d.pt. = Dermatophagoides pteronyssinus SD = Standardabweichung

mengefasst ein hochsignifikanter Unterschied $(p=0,0005)$ i. S einer Hemmung der Allergenprovokation (s. Tab. 3).

Zwischen den Allergenprovokationen $(\mathrm{P})$ bei Untersuchungsbeginn (Leerwert) sowie der Allergenprovokation ohne Montelukast im Verlauf der Untersuchung (P3 bei Gruppe I, P2 bei Gruppe II) zeigten sich keine nennenswerten Unterschiede, so dass die gewählte Provokationstestung als gut reproduzierbar anzusehen ist.

Wie sich aus Tab. 3 und Abb. 2 ablesen lässt, kam es in Gruppe I ausgehend von einer $\mathrm{PD}_{20 \text { Allergen }}$ von $16,6 \mathrm{BU}$ zu einem Anstieg auf 52,3 BU unter Montelukast mit Rückgang auf 31,9 BU nach 1 Tag Einnahmepause (-39\%). In der zweiten Gruppe fand sich eine Zunahme der $\mathrm{PD}_{20 A l l e r g e n}$ von $10 \mathrm{BU}$ auf 63,5 BU unter Montelukast, wobei nach 3-tägiger Einnahmepause der Wert bei 21,0 BU lag (-67\%) (Gruppe I: Vergleich Provokation (P)1, P2 und P4; Gruppe II: Vergleich P1, P3 und P4). Nur nach 3 Tagen war ein signifikanter Abfall $(\mathrm{p}=0,017)$ zu beobachten.

Die Messwerte der oszillatorischen Resistance wurden bei der Auswertung nicht berücksichtigt, da alle Patienten zuerst in der $\mathrm{FEV}_{1}$ um $20 \%$ abfielen, bevor sich die Resistance verdoppelte. 


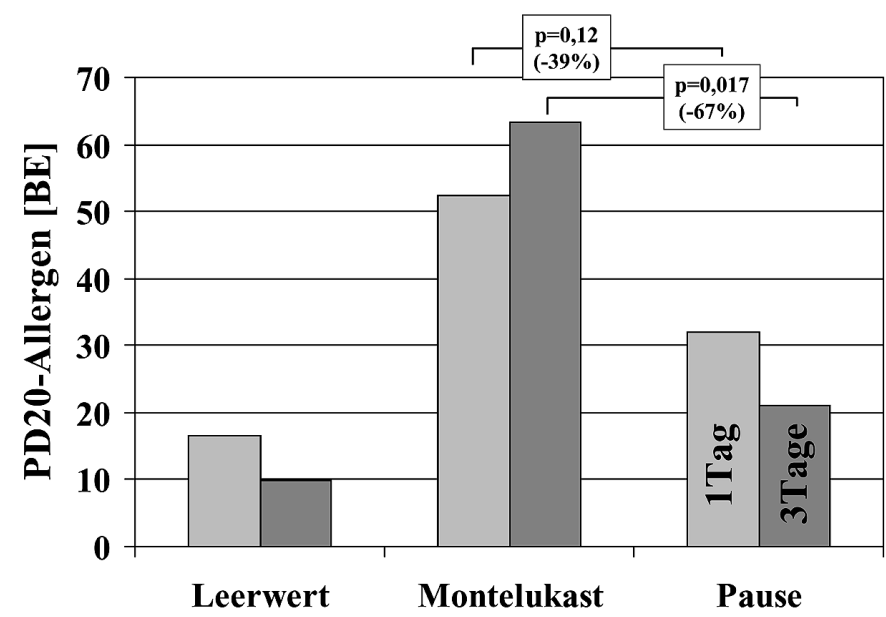

Abb. 2 PD20-Allergen nach 1 Tag (Gruppe 1) bzw. 3 Tage (Gruppe 2) Einnahmepause von Montelukast.

\section{Diskussion}

Von den insgesamt 16 Patienten mit Asthma zeigte sich in unserer Voruntersuchung [8] bei 12 Pat. ein unterschiedlich stark ausgeprägter protektiver Effekt auf die allergeninduzierte Sofortreaktion nach inhalativer Provokation $(p<0,0005)$. Die Auswirkungen auf die Spätreaktion wurden nicht untersucht. Die Allergeninhalationen wurden bewusst in dreiwöchigen Abständen durchgeführt, um eine Beeinflussung durch eine allergeninduzierte Steigerung der bronchialen Hyperreagibilität nach positiver Provokation auszuschließen [11]. Nach 1-tägiger Unterbrechung der Einnahme geht die $\mathrm{PD}_{20 \text { Allergen }}$ um $39 \%$ zurück; nach 3-tägiger Unterbrechung um $67 \%$. Erst dann ist der Unterschied signifikant $(p=0,017)$.

Das Ausmaß der Allergenprotektion ist im Einzellfall nicht vorauszusehen und unabhängig von der $\mathrm{PD}_{20 \text { Allergen vor Einnahme }}$ von Montelukast und auch unabhängig von dem verwendeten Allergen. Andererseits zeigen sich bei den „Montelukast-nonrespondern“ vor der Medikamenteneinnahme keine einheitlichen Befunde, die eine Voraussage ermöglichen. Für die inhalative Allergenprovokation bedeutet dies, dass im Einzelfall das Ausmaß der Unterdrückung der Bronchokonstriktion nicht vorauszusehen ist und z.T. erheblich höhere Allergendosen intrabronchial appliziert werden müssen, sofern Montelukast nicht vorher abgesetzt wurde. In welcher Zeit sich die Protektion zurückbildet, ist nach Literaturrecherchen und Nachfragen beim Hersteller bisher nicht untersucht worden.

Um einen Einfluss der erlaubten Bedarfsmedikation (kurzwirksame $\beta$-Mimetika und Anticholinergika) auf die Provokationstestung zu vermeiden, haben wir die in der Leitlinie zur Allergenprovokation [9] vorgeschlagenen Mindestabsetzfristen eingehalten; kurzwirksame $\beta$-Mimetika: 6 Stunden, Anticholinergika: 12 Stunden.

Inhalative Kortikosteroide beeinflussen fast nur die Spätreaktion nach Allergenprovokation, so dass wir diese Medikation in konstanter Dosierung während des Untersuchungszeitraumes erlaubt haben.
Die erste Publikation, die sich unseres Wissens nach mit dem Effekt von Montelukast auf die inhalative Allergenprovokation beschäftigt hat, stammt von Diamant u. Mitarb. aus dem Jahre 1999 [12]. Es wurde in einer doppelblind, plazebokontrollierten Crossover-Studie bei 12 männlichen Asthmatikern u.a. die Auswirkung einer Montelukastgabe 36 und 12 Stunden vor Allergenprovokation mit Hausstaubmilben (Dermatophagoides pteronyssinus) überprüft. Hierbei zeigte sich eine signifikante Hemmung der allergeninduzierten Sofort- und Spätreaktion durch die zweimalige Einnahme von Montelukast. Somit findet sich eine Übereinstimmung mit unseren Ergebnissen.

In einer anderen Arbeit untersuchten Phipatanakul u. Mitarb. den Effekt einer einwöchigen Montelukast-Medikation im Vergleich zu Plazebo bei 18 katzenallergischen Kindern im Alter zwischen 6 und 14 Jahren bei einer einstündigen Exposition gegenüber Katzen [13]. Es kam unter Montelukast zu einem signifikant geringeren Abfall der $\mathrm{FEV}_{1}$, während die Symptome des oberen Respirationstraktes nicht signifikant beeinflusst wurden.

Zusammenfassend ziehen wir aus unserer Untersuchung den Schluss, dass Montelukast mindestens 3, besser 5 Tage vor inhalativer bronchialer Allergenprovokation abgesetzt werden sollte.

\section{Literatur}

${ }^{1}$ Jarvis B, Markham A. Montelukast. A review of its therapeutic potential in persistent asthma. Drugs 2000; 59: 891 - 928

${ }^{2}$ Holgate ST, Sampson AP. Antileucotriene therapy: future directions. Am J Respir Crit Care Med 2000; 161 Suppl: 125-132

3 Pizzchini E, Leff JA, Reiss TF et al. Montelukast reduces eosinophilic inflammation in asthma: a randomized, controlled trial. Eur Respir J 1999; $14: 12-18$

${ }^{4}$ Drazen JM, Israel E, O'Bourne PM. Treatment of asthma with drugs modifying the leucotriene pathway. N Engl J Med 1999; 21, 340: 197-206

${ }^{5}$ Knorr B, Matz J, Bernstein J et al. Montelukast for chronic asthma in 6 to 14-year-old children.A randomized, double-blind trial. JAMA 1998; 279: $1181-1186$

${ }^{6}$ Leff JA, Busse WW, Pearlman D et al. Montelukast,a leucotriene-receptor antagonist, for the treatment of mild asthma and exercise-induced bronchocostriction. N Engl J Med 1998; 339: 147-152

${ }^{7}$ Robinson DS, Campbell D, Barnes PJ. Addition of leukotriene antagonists to therapy in chronic persistent asthma: a randomized doubleblind placebo-controlled trial. Lancet 2001; 357: 2007-2011

${ }^{8}$ Riffelmann FW, Droste D, Lauter $\mathrm{H}$ et al. Einfluss von Montelukast auf die inhalative bronchiale Allergenprovokation. Pneumologie 2002; 56: $493-497$

${ }^{9}$ Gonsior E, Henzgen M, Jörres RA et al. Leitlinie für die Durchführung bronchialer Provokationstests mit Allergenen. Pneumologie 2002; 56: $187-198$

${ }^{10}$ Köhler D, Fleischer W. Theorie und Praxis der Inhalationstherapie. München: Arcis, 2000

${ }^{11}$ Ciprandi G, Ricca V, Landi M et al. Allergen-specific nasal challenge: response kinetics of clinical and inflammatory events to rechallenge. Int Arch Allergy Immunol 1998; 115: 157-161

${ }^{12}$ Diamant Z, Grootendorst DC, Veselic-Charvat M et al. The effect of montelukast (MK-0476), a cysteinyl leucotriene receptor antagonist, on allergen-induced airway responses and sputum cell counts in asthma. Clin Exp Allergy 1999; 29: $42-51$

13 Phipatanakul W, Nowak-Wegrzyn A, Eggleston PA et al. The efficacy of montelukast in the treatment of cat allergen-induced asthma in children. J Allergy Clin Immunol 2002; 109: 794-799 